\title{
(6) OPEN ACCESS \\ The Addenbrooke's Cognitive Examination for the differential diagnosis and longitudinal assessment of patients with parkinsonian disorders
}

\author{
Timothy Rittman, Boyd C Ghosh, Peter McColgan, David P Breen, Jonathan Evans, \\ Caroline H Williams-Gray, Roger A Barker, James B Rowe
}

Department of Clinical Neurosciences, University of Cambridge, Cambridge, UK

\section{Correspondence to} Dr Timothy Rittman, Department of Clinical Neurosciences, University of Cambridge, Herchel Smith Building, Forvie Site, Addenbrooke's Hospital Site, Robinson Way, Cambridge CB2 OSZ, UK;

tr332@medschl.cam.ac.uk

Received 6 August 2012 Revised 28 November 2012 Accepted 30 November 2012 Published Online First 9 January 2013

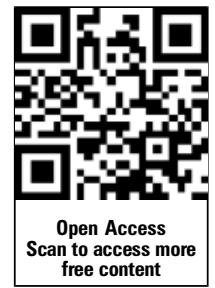

To cite: Rittman $\mathrm{T}$ Ghosh BC, McColgan P, et al. I Neurol Neurosurg Psychiatry 2013:84: 544-551.

\section{ABSTRACT \\ Objective Differentiating idiopathic Parkinson's disease from atypical parkinsonian syndromes is challenging, especially in the early stages. We assessed whether the Revised Addenbrooke's Cognitive Examination (ACE-R) could differentiate between parkinsonian syndromes and reflect longitudinal changes in cognition in these disorders.}

Methods The ACE-R was administered at baseline and after approximately 18 months to 135 patients with parkinsonian disorders: 86 with idiopathic Parkinson's disease (PD), 30 with progressive supranuclear palsy (PSP), 19 with corticobasal degeneration (CBD). We assessed differences between groups for ACE-R, ACE-R subscores and Mini Mental State Examination (MMSE) scores at baseline (analyses of variance, receiver operating characteristics curves), and the interaction between diagnosis and change in ACE-R scores between visits (analyses of variance).

Results The ACE-R verbal fluency subscore distinguished between PSP and PD with a high sensitivity (0.92) and specificity (0.87); total ACE-R score and the visuospatial subscore were less specific $(0.87$ and 0.84 respectively) and sensitive (0.70 and 0.73 ). Significant group level differences were found between PD and PSP for MMSE and ACE-R (total score and subscores for attention and concentration, fluency, language, and visuospatial function), and between PD and CBD for the ACE-R visuospatial subscore.

Performance worsened between visits for ACE-R score in $P D(p=0.001)$ and $C B D(p=0.001)$; visuospatial subscore in PD ( $p=0.003)$, PSP $(p=0.022)$ and CBD $(p=0.0002)$; and MMSE in CBD $(p=0.004)$.

Conclusions We propose the ACE-R, particularly the verbal fluency subscore, as a valuable contributor to the differential diagnosis of parkinsonian syndromes in the correct clinical context. The ACE-R may reflect disease progression in $\mathrm{PD}$ and $\mathrm{CBD}$.

\section{INTRODUCTION}

Idiopathic Parkinson's disease (PD) can be difficult to distinguish from atypical parkinsonian syndromes such as progressive supranuclear palsy (PSP) and corticobasal degeneration (CBD), especially in the early stages of disease. A simple test that is easy to administer, differentiates between parkinsonian disorders and identifies decline over time would be a welcome addition to the clinical and research armoury. For clinicians such a test could reduce the high rate of uncertainty, diagnostic delay and misdiagnosis, which are especially common for PSP.

In research, the urgent need for new disease measures and better diagnostic differentiation is highlighted by current progress toward neuroprotective and disease-modifying therapies: early diagnosis and the ability to monitor progression will be increasingly important with disease-specific treatments. ${ }^{1}$ A perfect single test may not exist, but a combination of clinical, cognitive and biological tests, as biomarkers of disease, would provide a significant step forward. ${ }^{2}$ In this study, we consider the potential role of cognitive tests for these purposes.

PD, PSP and CBD are all movement disorders with varying patterns of cognitive impairment. $\mathrm{PD}$ is characterised by asymmetric bradykinesia, with tremor, rigidity and/or postural instability. While frontostriatal executive impairments are common in PD, an early dementia is uncommon and tends to be heralded by deficits in posterior cortically based cognitive functions. ${ }^{3}$ PSP typically presents with symmetrical akinesia and rigidity, early falls and a supranuclear gaze palsy. It is associated with a frontal dysexecutive syndrome, impaired fluency, disinhibition and cognitive inflexibility, together with impairments in language, motivation, emotional and social cognition..$^{4-6}$ CBD is highly variable, but includes the alien limb syndrome, asymmetric dystonia, apraxia, myoclonus, non-fluent aphasia and visuospatial impairment. ${ }^{7}$ Cognitive involvement in CBD ranges from near normal cognition to severe dementia, although language syndromes such as non-fluent aphasia are common. ${ }^{6} 8$

Despite characterisation of individual cognitive profiles, the difference between diseases has been less well studied. Cognitive tests have been suggested to differentiate between PD and atypical parkinsonism. ${ }^{9}{ }^{10}$ A small study using a battery of executive tests, including verbal fluency, correctly identified 15 of 16 patients with PSP from those with PD or multiple system atrophy, but with wide CIs. The Revised Addenbrooke's Cognitive Examination (ACE-R) and verbal fluency have been found to distinguish between atypical parkinsonian disorders and Alzheimer's disease. ${ }^{11}$ However, this is rarely a clinical conundrum; the more common clinical dilemma is one of differentiating PD from other parkinsonian disorders.

Imaging techniques have also been used to distinguish between PD and atypical parkinsonism. Typical signs such as the 'humming bird' and 
'mickey mouse' signs may occur in PSP while formal quantification of structural MRI changes using voxel-based morphometry have suggested patterns of atrophy that may help distinguish between PD and $\mathrm{PSP}^{12}$ with a specificity of $84 \%$ and sensitivity of $79 \%$ based on atrophy in the cerebellar peduncles and midbrain. ${ }^{13}$ Contrasting atrophy patterns are also seen between CBD and PSP; presence of midbrain atrophy has a specificity of $96.2 \%$ and sensitivity of $93.8 \%$ to distinguish PSP from CBD, and from asymmetrical cortical atrophy a specificity of $87.5 \%$ and sensitivity of $100 \% .{ }^{14} \mathrm{~A}$ more quantitative approach was less successful, achieving a positive predictive value (PPV) of $66.7 \%$ for PSP and $71.4 \%$ for CBD by measuring changes in the midbrain, pontine tegementum and left frontal eye field. ${ }^{15}$

Given the cost and limited success of brain imaging to distinguish PD from other parkinsonian syndromes, attention has turned to simpler neuropsychological measures. The ACE-R is a comprehensive and easily administered pen and paper test of cognition which has been used in a number of neurodegenerative diseases, including PD, Alzheimer's disease ${ }^{16}$ and frontotemporal dementia. ${ }^{17}$ It consists of a 100 -point test divided into five cognitive subdomains (table 1).

Based on the differing published descriptions of cognitive profiles between diseases, we set out to assess whether the ACE-R, the ACE-R subscores or the widely used Mini Mental State Examination (MMSE) might be useful in two distinct ways. First, whether these cognitive tests distinguish between PD, PSP and CBD, at a group average level and in terms of receiver operator characteristic (ROC) curves. Second, whether these tests have the potential to monitor disease progression over time in these disorders.

\section{METHODS}

\section{Patient recruitment}

We recruited patients with PD $(n=86)$, PSP $(n=30)$ and CBD $(n=19)$. Patients with PD were newly diagnosed and recruited as part of a community-based incidence study of PD in Cambridgeshire (PICNICS study). All PICNICS study participants

Table 1 The ACE-R test multiple cognitive domains, summed to a score of 100 points

\begin{tabular}{|c|c|c|}
\hline Cognitive domain & Abilities tested & Score \\
\hline \multirow[t]{4}{*}{ Attention and concentration } & Orientation & 18 \\
\hline & Registration & \\
\hline & Attention and concentration & \\
\hline & Recall & \\
\hline \multirow[t]{4}{*}{ Memory } & Anterograde memory (name and address) & 26 \\
\hline & Retrograde memory & \\
\hline & Recall (long delay) & \\
\hline & Recognition & \\
\hline \multirow[t]{2}{*}{ Verbal fluency } & Letter fluency ( $p$-words) & 14 \\
\hline & Category fluency (animals) & \\
\hline \multirow[t]{4}{*}{ Language } & Comprehension & 26 \\
\hline & Repetition & \\
\hline & Naming & \\
\hline & Semantic knowledge & \\
\hline \multirow[t]{3}{*}{ Visuospatial } & Copying drawings & 16 \\
\hline & Drawing a clock face & \\
\hline & Perception & \\
\hline $\begin{array}{l}\text { The MMSE is extracted as a } 3 \\
\text { ACE-R, Addenbrooke's Cogniti } \\
\text { Examination. }\end{array}$ & $\begin{array}{l}\text {-point score from elements of the ACE-R. } \\
\text { e Examination Revised; MMSE, Mini-Mental Sta }\end{array}$ & \\
\hline
\end{tabular}

who had returned for a follow-up assessment at the time of data analysis were included in this study, representing $37 \%$ of the overall PICNICS study population. Patients were recruited from primary and secondary care and assessed using a battery of cognitive and neurological tests, in addition to a semi-structured interview. All patients fulfilled Queen Square Brain Bank criteria for PD. Patients with PSP and CBD were recruited from a regional tertiary referral specialist clinic at the Cambridge University Hospitals NHS Trust (Addenbrookes), UK. This clinic encourages regional referral of all cases of PSP and CBD, from movement disorders specialists and memory clinics. It is not restricted to those with dementia or cognitive impairment. Standard diagnostic criteria were applied for PSP $^{18}$ and $\mathrm{CBD}^{8}$ Of nine PSP patients who proceeded to post mortem, all had neuropathological confirmation of PSP. All patients gave written, informed consent and ethical approval was obtained from the Cambridge 2 Research Ethics Committee, Cambridge, UK.

\section{Data collection}

Cognitive assessment using the ACE-R and MMSE was performed on two separate occasions, at a baseline visit and again at a follow-up visit. For patients with PD, a planned visit at approximately 18 months was arranged as part of the PICNICS study. For patients with PSP and CBD, the clinic visit closest to 18 months follow-up was identified. Assessment was carried out in a quiet room by investigators familiar with the ACE-R. Collection of data in patients with PSP and CBD began before widespread use of the Movement Disorder Society Unified Parkinson's Disease Rating Scale (MDS-UPDRS); therefore motor function was assessed using the original UPDRS III, whereas patients with PD were evaluated with the MDS-UPDRS III.

\section{Statistical analysis}

Between-group differences at baseline were investigated using a one-way analysis of variance (ANOVA). Follow-up scores were normalised to a time interval of 18 months. Group difference for change in scores between visits was assessed using a multiple measures ANOVA with fixed and random effects. If appropriate (as indicated in the results section), significance values were Bonferroni corrected for multiple comparisons. All statistical analyses were performed in $\mathrm{R}$ statistical software (V.2.14.1; http://cran.r-project.org/).

ROC curves and associated statistics were generated using the pROC package. ${ }^{19}$ An optimal threshold for each cognitive test per pairwise comparison was identified using a 'closest top-left' algorithm: CIs were obtained for the area under the curve (AUC), sensitivity and specificity by a stratified bootstrap analysis with 5000 permutations. Sensitivity was defined as the ratio between true positives and all those who carried a diagnosis of interest (condition positive) for a given threshold. Specificity was defined as the proportion of false negatives among all those who did not carry the diagnosis of interest (condition negative) for a given threshold. PPV was defined as the ratio between the number of true positive subjects and the total number of positive results (false positive and true positives) for each diagnosis at a given threshold; Negative predictive value (NPV) was defined as the ratio between the number of true negative results and the total number of negative results for each diagnosis at a given threshold.

\section{RESULTS}

\section{Demographics}

Demographic details are shown in table 2. Patients with CBD were younger than those with $\operatorname{PD}(p=0.004)$ and those with 
Table 2 Demographics for the study population recorded at the baseline visit

\begin{tabular}{lllll}
\hline & PD & PSP & CBD & p \\
\hline Age (years) & 69.0 & 70.9 & 62.0 & 0.005 \\
Gender (men/women) & $58 / 30$ & $13 / 17$ & $10 / 9$ & 0.2 \\
Handedness (right/left/ambidextrous) & $74 / 13 / 1$ & $29 / 1$ & $16 / 3$ & 0.5 \\
Years of education & 11.9 & 12.0 & 13.0 & 0.3 \\
UPDRS III (on therapy) & - & 29.7 & 29.0 & 0.8 \\
MDS-UPDRS III (on therapy) & 30.5 & - & - & - \\
$\begin{array}{l}\text { Duration of symptoms at baseline (years) } \\
\text { Percentage on dopaminergic therapy }\end{array}$ & 1.8 & 3.2 & 3.1 & $<0.0001$ \\
$\begin{array}{l}\text { Mean levodopa dose of those on } \\
\text { medication (mg) }\end{array}$ & 27.3 & 18.1 & 30 & 0.05 \\
\hline
\end{tabular}

$\mathrm{p}$ Values based on $\mathrm{F}$ tests in one-way analysis of variance, or $\chi^{2}$, as appropriate. CBD, corticobasal degeneration; PD, Parkinson's disease; PSP, progressive supranuclear palsy.

PSP $(p=0.001)$. PSP and PD groups did not differ in age $(p=0.3)$. The duration of symptoms reported by the patient or carer at the first clinical assessment was shorter for patients with
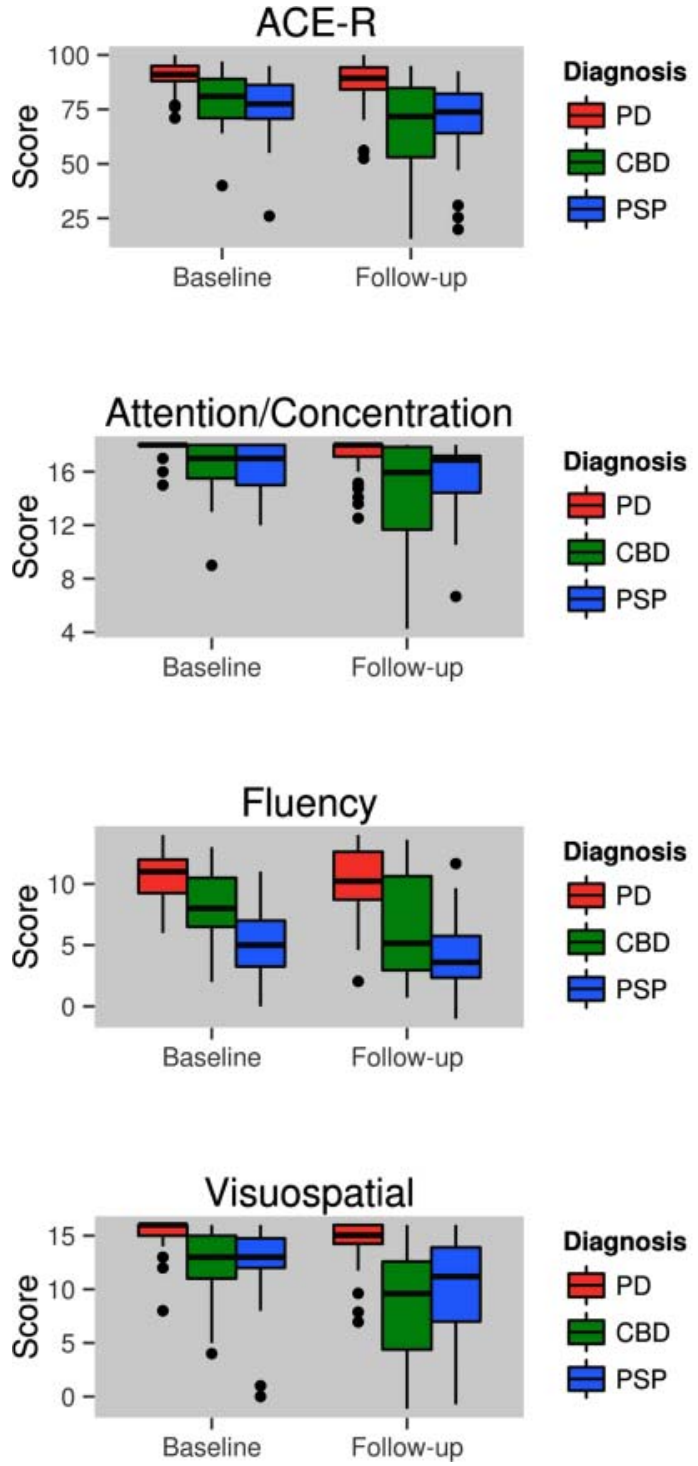

PD compared with those with PSP $(\mathrm{p}=0.003)$ and CBD $(p<0.001)$. There were no significant differences between groups in terms of baseline education, handedness or gender. ACE-R scores and subscores for each group are shown in figure 1. There was a trend toward more patients with PD taking levodopa than the other two groups, although those that did took a similar dose. Patients with PD were seen for follow-up on average at 19.0 months, slightly later than PSP (16.1 months, $\mathrm{p}=0.007)$ or CBD (14.3 months, $\mathrm{p}=<0.001)$. Cognitive scores at baseline and follow-up are summarised in figure 1. The selection of patients with PD matched the demographics of the whole PICNICS study population in terms of age, gender, handedness, years of education, MDS-UPDRS III, levodopa equivalent dose and ACE-R score ( $p>0.1$ in all cases).

Diagnostic differentiation at baseline

At the baseline visit, a between-group comparison showed a significant effect of diagnosis on the ACE-R score for attention and concentration, fluency, language and visuospatial subscores, but not for memory subscores (table 3 ).

To investigate the effect of disease duration and delayed diagnosis between groups, we took advantage of a feature in
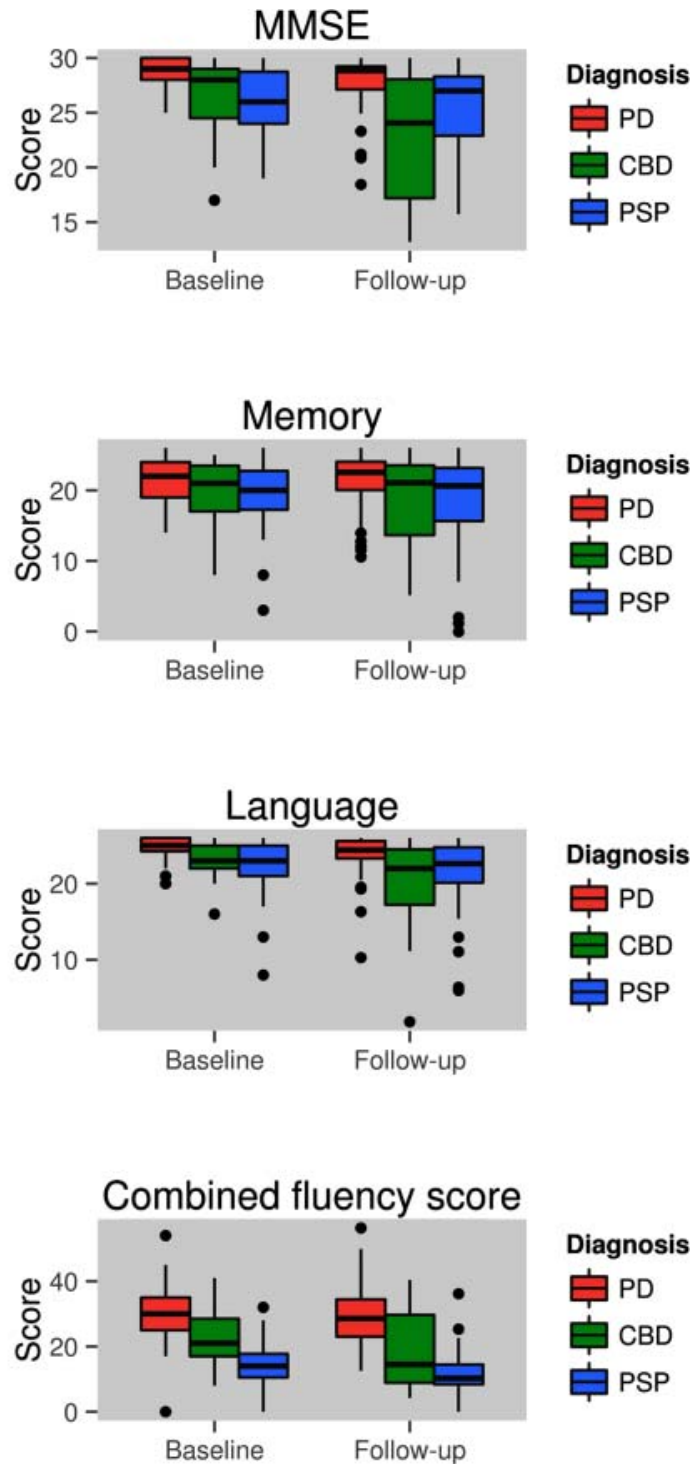

Figure 1 ACE-R scores and subscores at baseline and follow-up visits for patients with PD, PSP and CBD. ACE-R, Addenbrooke's Cognitive Examination Revised; CBD, corticobasal degeneration; MMSE, Mini Mental State Examination; PD, Parkinson's disease; PSP, progressive supranuclear palsy. 
Table 3 Between group differences at baseline visit for ACE-R score and subscores, and combined raw verbal fluency (combined letter and category fluencies)

\begin{tabular}{|c|c|c|c|c|c|c|}
\hline & \multirow[b]{2}{*}{ DF } & \multirow[b]{2}{*}{$\mathbf{F}$} & \multirow[b]{2}{*}{$\mathbf{p}$} & \multicolumn{3}{|c|}{ Post hoc $t$ test $p$ values } \\
\hline & & & & $\begin{array}{l}\text { PD vs } \\
\text { PSP }\end{array}$ & $\begin{array}{l}\text { PD vs } \\
\text { CBD }\end{array}$ & $\begin{array}{l}\text { PSP vs } \\
\text { CBD }\end{array}$ \\
\hline ACE-R & 2 & 18.4 & $<0.0001$ * & $<0.0001^{*}$ & 0.08 & ns \\
\hline MMSE & 2 & 18.6 & $<0.0001^{*}$ & $0.002^{*}$ & ns & ns \\
\hline $\begin{array}{l}\text { Attention/ } \\
\text { concentration }\end{array}$ & 2 & 28.6 & $<0.0001^{*}$ & 0.01 * & ns & ns \\
\hline Memory & 2 & 3.4 & ns & & & \\
\hline Fluency & 2 & 51.7 & $<0.0001^{*}$ & $<0.0001^{*}$ & 0.07 & $0.02^{*}$ \\
\hline Language & 2 & 13.9 & $<0.0001^{*}$ & 0.01 * & ns & ns \\
\hline Visuospatial & 2 & 0.5 & $<0.0001^{*}$ & $0.005^{*}$ & $0.02^{*}$ & ns \\
\hline $\begin{array}{l}\text { Combined fluency } \\
\text { score }\end{array}$ & 2 & 30.64 & $<0.0001^{*}$ & $<0.0001^{*}$ & ns & $0.04^{*}$ \\
\hline \multicolumn{7}{|c|}{$\begin{array}{l}\text { Post hoc } t \text { tests are shown wherever analysis of variance showed a significant group } \\
\text { difference. } \\
\text { *Significant Bonferroni corrected } p \text { values }<0.05 \text {. ns=not significant, } p>0.1 \text {. } \\
\text { ACE-R, Addenbrooke's Cognitive Examination Revised; CBD, corticobasal } \\
\text { degeneration; MMSE, Mini Mental State Examination; PD, Parkinson's disease; } \\
\text { PSP, progressive supranuclear palsy. }\end{array}$} \\
\hline
\end{tabular}

our data, that the time from first reported symptom in the PD group at follow-up (3.3 years) was not significantly different from the baseline symptom duration in the PSP (3.2 years) and CBD (3.1 years) groups. Hence, baseline PSP and CBD scores and follow-up PD scores were at similar symptom duration, and could be compared directly. ANOVA results for this comparison showed a similar pattern of significance in group differences, except that pairwise comparison of visuospatial scores were non-significant between PD and PSP $(p=0.1)$, and between PD and CBD $(p=0.2)$.

To investigate the predictive accuracy of each score for individual subjects, we performed ROC curve analysis (figure 2). Detailed results are shown in table 3 for all ACE-R scores and subscores where the AUC exceeded 0.85, and for the MMSE as a comparison. Best differentiation was seen between PD and PSP subjects, in particular the verbal fluency subscore. Subdividing the fluency score into letter and category components showed a diagnostic differentiation between PD and PSP similar to the combined scaled score using letter fluency alone (threshold 7 words per minute, AUC 0.93 , specificity 0.85 , sensitivity 0.83 , PPV 0.81, NPV 0.93), and slightly poorer differentiation using category fluency (threshold 13.5 words per minute, AUC 0.89, specificity 0.81 , sensitivity 0.83 , PPV 0.63 , NPV 0.93 ).

\section{Measuring change over time}

An interaction between diagnosis and change in score between visits was seen in the ACE-R total score, visuospatial subscore, attention and concentration subscore, and MMSE (table 4). Post hoc $t$ tests showed that ACE-R total scores declined significantly in PD and CBD (see table 5). The decline was greatest in CBD. There was greatest decline in visuospatial subscores in the CBD group, although the PD and PSP groups also showed a significant fall. Only the CBD group showed a significant decline in MMSE between visits. Analysis of follow-up scores without normalisation for interval to follow-up showed an almost identical pattern of significance, the only difference being a marginally significant difference for ACE-R score between visits in the PSP group $(p=0.04)$.

\section{DISCUSSION}

Our data show that the ACE-R, and in particular its total score, verbal fluency and visuospatial subscores, distinguish between PD and PSP, and to a lesser extent between PD and CBD. The differentiation between PD and PSP using verbal fluency alone (specificity 0.92 , sensitivity 0.87 ) outperforms previous reports of diagnostic differentiation achieved using imaging methods alone. $^{12}{ }^{13}$ In addition, our data suggest that the ACE-R may be helpful for tracking disease progression over time, particularly in CBD. These findings suggest that in the appropriate clinical context, the ACE-R may provide useful information in the diagnosis and tracking of parkinsonian disorders.

The good PPVs and NPVs, low cost and simplicity of these cognitive biomarkers makes them especially useful when the cost and expertise required for routine clinical MRI or positron emission tomography is prohibitive. This includes many regions of the world where imaging is not available. ${ }^{20}$ Cognitive tests have the potential of being safe, inexpensive and low-tech additions to the diagnostic armoury that can be used in a range of clinical settings-primary, secondary or tertiary care and research studies. The ACE-R has even been validated in a number of languages, although our findings would require replication in different populations to properly assess generalisation. ${ }^{17} 2122$

Different parts of the cognitive assessment had different value in the differentiation of PD, PSP and CBD. We focused on the ACE-R rather than the MMSE, as it was superior in differentiating disorders (see ROC curves, figure 2) and was more sensitive to decline over time. The role of verbal fluency was particularly striking. Fluency deficits have been described in $\mathrm{PD}^{23} \mathrm{PSP}^{5} 24$ and CBD. ${ }^{25}$ The decline in fluency is not entirely due to bradykinesia, as fluency requires very little motor function. Interestingly, patients with PSP or CBD may generate a small number of low-frequency words (eg, 'peramubulator') rather than high-frequency words (eg, 'put', 'people'). This is supported by evidence from functional imaging suggesting that verbal fluency is associated with activation of a diffuse cortical network $^{26}$ but with an emphasis on Brodmann area 45 for lexical retrieval and Brodmann area 46 in phonological processing during word generation. ${ }^{27}$ Patients with brain lesions in the left dorsolateral frontal lobe were found to have impaired verbal fluency if there were additional or solitary striatal lesions. ${ }^{28}$ Neuropathology studies confirm that both of these sites are affected in PSP and CBD. ${ }^{29}{ }^{30}$ Verbal fluency impairment has previously been reported in the early stages of $\mathrm{PD},{ }^{3}$ and is consistent with the Braak staging hypothesis that predicts frontal lobe cortical pathology. ${ }^{31}$

Changes in higher visual processing are present in all three disorders, but take different forms in each. Patients with PD may experience hallucinations or misperceptions-usually later in the disease process-that may be related to medication side effects or impaired attentional processing. ${ }^{32}$ Patients with CBD and, to a lesser extent, those with PSP perform poorly on visuospatial tasks. Patients with PSP are impaired more with 'object decision' tasks, thought to be related to impairment in the ventral stream of visuospatial processing, whereas patients with CBD are typically worse on spatial location tasks, thought to relate to the parietal atrophy disrupting the dorsal stream of visuospatial processing. ${ }^{33}$ These previous findings are in broad agreement with our results. The ACE-R visuospatial tests rely more on spatial abilities than object recognition and is therefore likely to be more sensitive to the changes seen in CBD and PSP, but is less likely to detect the misperceptions more commonly experienced in PD. 
Figure 2 Receiver operating characteristics curves distinguishing between diagnostic groups using baseline ACE-R scores and subscores. Thresholds were chosen using a 'top left corner' algorithm. Cls are shown for sensitivity and specificity. ACE-R, Addenbrooke's Cognitive Examination Revised; AUC, area under the curve; $\mathrm{CBD}$, corticobasal degeneration; MMSE, Mini Mental State

Examination; PD, Parkinson's disease; PSP, progressive supranuclear palsy.
ACE-R Total

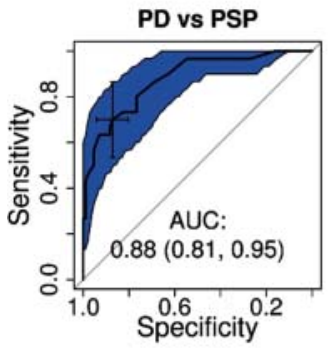

PD vs CBD
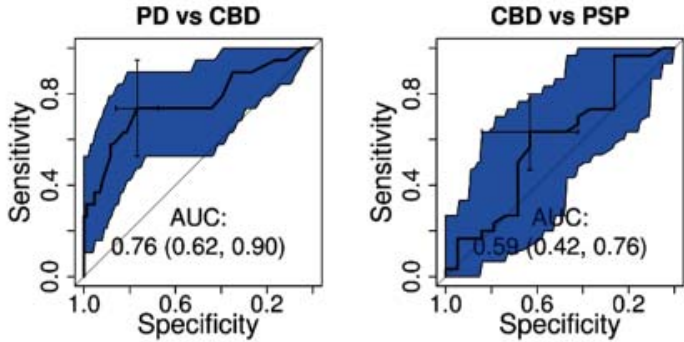

MMSE
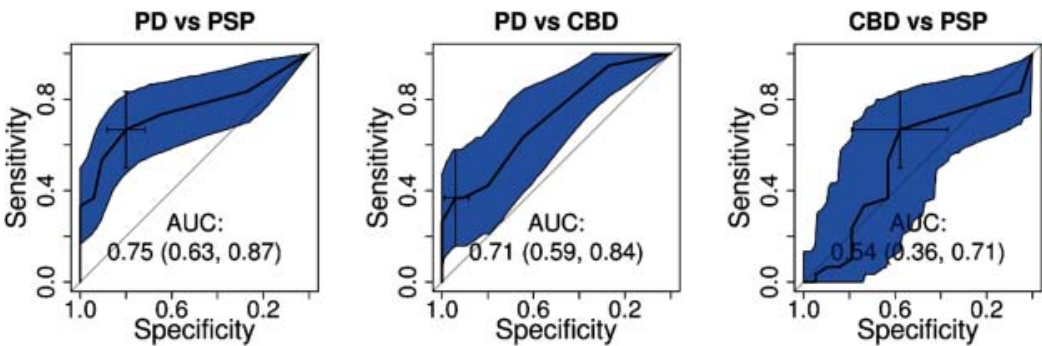

Fluency Subscore
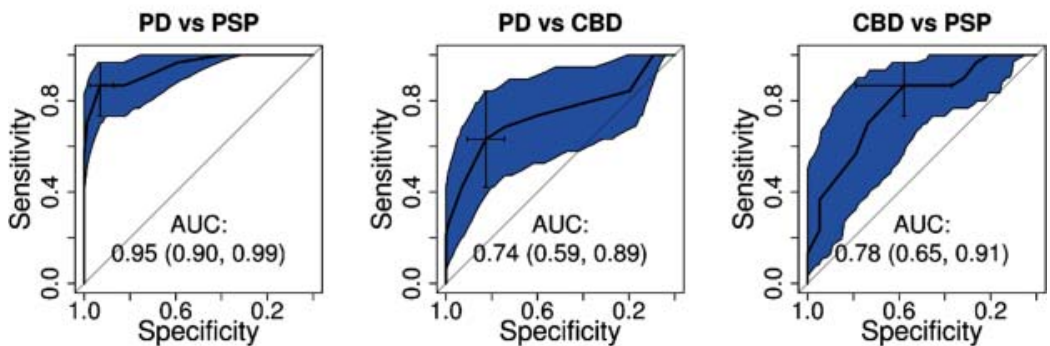

\section{Combined Fluency Score}
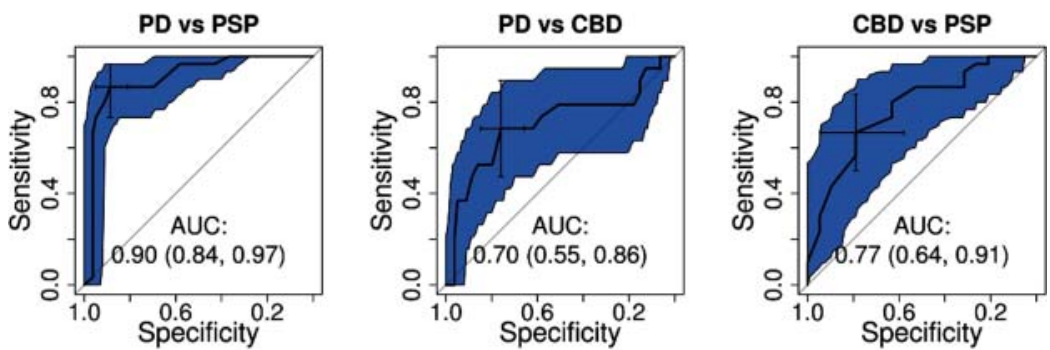

\section{Visuospatial Subscore}

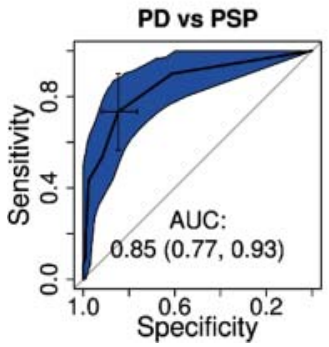

Statistically significant group differences in test scores do not necessarily indicate clinically meaningful differences in test scores, especially in large studies. For many of our results, we suggest that the differences between groups and over time are both statistically significant and clinically meaningful. MMSE scores declined by 2.7 points in the CBD group, which is close to the SD of 2.8 found in a large dementia population, ${ }^{34}$ suggesting this change is clinically significant. Large absolute differences were seen in PSP and CBD groups for total ACE-R score (PSP 6.0, CBD 10.9, of maximum score 100) and visuospatial subscores (PSP 2.5, CBD 3.8, of maximum 16 points). Although we do not have functional rating scales or measures of activities 
Table 4 ROC characteristics for between diagnostic group comparisons at optimal thresholds, determined using a 'closest top left' algorithm

\begin{tabular}{|c|c|c|c|c|c|}
\hline & Threshold & Specificity $(95 \% \mathrm{Cl})$ & Sensitivity (95\% Cl) & PPV & NPV \\
\hline \multicolumn{6}{|l|}{ PSP vs PD } \\
\hline ACE-R & 83.5 & 0.87 (0.80 to 0.94$)$ & $0.70(0.53$ to 0.87$)$ & 0.66 & 0.89 \\
\hline MMSE & 27.5 & 0.80 (0.72 to 0.88 ) & 0.67 (0.50 to 0.83 ) & 0.54 & 0.87 \\
\hline Fluency & 7.5 & 0.93 (0.87 to 0.98 ) & 0.87 (0.73 to 0.97$)$ & 0.81 & 0.95 \\
\hline Combined raw fluencies & 20.5 & 0.89 (0.81 to 0.95$)$ & 0.87 (0.73 to 0.97 ) & 0.74 & 0.95 \\
\hline Visuospatial & 14.5 & 0.84 (0.77 to 0.92 ) & 0.73 (0.57 to 0.87 ) & 0.63 & 0.90 \\
\hline \multicolumn{6}{|l|}{ CBD vs PD } \\
\hline ACE-R & 87 & $0.77(0.67$ to 0.85$)$ & $0.74(0.53$ to 0.89$)$ & 0.41 & 0.93 \\
\hline MMSE & 25.5 & 0.94 (0.88 to 0.99$)$ & 0.37 (0.16 to 0.58$)$ & 0.58 & 0.87 \\
\hline Fluency & 8.5 & 0.83 (0.74 to 0.91$)$ & 0.63 (0.42 to 0.84$)$ & 0.44 & 0.91 \\
\hline Combined raw fluencies & 24.5 & 0.76 (0.66 to 0.84 ) & 0.68 (0.47 to 0.89 ) & 0.40 & 0.91 \\
\hline Visuospatial & 13.5 & 0.92 (0.85 to 0.98 ) & 0.68 (0.47 to 0.89 ) & 0.65 & 0.93 \\
\hline \multicolumn{6}{|l|}{ PSP vs CBD } \\
\hline ACE-R & 79.5 & 0.63 (0.42 to 0.84 ) & 0.63 (0.47 to 0.80$)$ & 0.73 & 0.52 \\
\hline MMSE & 27.5 & 0.58 (0.37 to 0.79 ) & 0.67 (0.5 to 0.83 ) & 0.71 & 0.52 \\
\hline Fluency & 7.5 & 0.58 (0.37 to 0.79 ) & 0.87 (0.73 to 0.97 ) & 0.81 & 0.97 \\
\hline Combined raw fluencies & 16.5 & 0.79 (0.58 to 0.95$)$ & 0.67 (0.5 to 0.83 ) & 0.83 & 0.60 \\
\hline Visuospatial & 10.5 & $0.26(0.11$ to 0.47$)$ & 0.9 (0.8 to 1.0$)$ & 0.70 & 0.45 \\
\hline
\end{tabular}

Corresponding ROC curves are shown in figure 2.

ACE-R, Addenbrooke's Cognitive Examination Revised; CBD, corticobasal degeneration; MMSE, Mini Mental State Examination; NPV, negative predictive value; PD, Parkinson's disease; PPV, positive predictive value; PSP, progressive supranuclear palsy; ROC, receiver operating characteristics.

of daily living, we suggest that the magnitudes of these changes are likely to be clinically significant. In contrast, the PD group showed much smaller changes in total ACE-R score (1.9 points) and the visuospatial subscore ( 0.5 points), which lie within the range of test variation (inter-rater variance and test-retest) and are less likely to be clinically meaningful. In the context of PD, it has recently been demonstrated that the ACE-R reflects functional changes; the authors could identify the difference between cognitively well patients with PD and those with PD and mild cognitive impairment using a cut-off of 89 (specificity of $84 \%$, sensitivity $69 \%) .{ }^{35}$ Our findings would support the use of cognitive measures in diagnostic criteria, although neuropathological correlation would be required to confirm this.

In comparing groups in this study, we are reliant on clinical diagnostic criteria, which may not always reflect underlying pathology. Clinical diagnostic criteria for $\mathrm{PSP}^{18}$ tend to be specific, with a diagnostic accuracy of $92 \%$ in patients meeting criteria. ${ }^{36}$ They may be insensitive to PSP pathology in patients with a PD-like syndrome ${ }^{37}$ or no clinical disease. ${ }^{38}$ In our cohorts, many patients with PSP had consented to brain donation in a regional brain bank, providing neuropathological confirmation in nine out of nine cases, in keeping with the high clinicopathological correlations in other centres.

There are several current clinical diagnostic criteria for CBD, recently reviewed by Mathew et al. ${ }^{8}$ They applied clinical criteria in early CBD, finding sensitivities of $23.5-67.5 \%$, increasing to $87.5-92.5 \%$ in later stage disease depending on the criteria applied. Diagnostic uncertainty is further reflected in the ante-mortem misclassification of CBD from post-mortem studies. ${ }^{3940}$ Our findings therefore support the use of cognitive measures in diagnostic criteria for PD, PSP and CBD, although further neuropathological correlation would be helpful to

Table 5 Group differences for the change in ACE-R and subscores between baseline and follow-up visit scores, normalised to a rate of change over 18 months

\begin{tabular}{|c|c|c|c|c|c|c|c|c|c|c|c|c|}
\hline & \multirow[b]{3}{*}{ DF } & \multirow[b]{3}{*}{$\mathbf{F}$} & \multirow[b]{3}{*}{$\mathbf{p}$} & \multicolumn{9}{|c|}{ Mean scores at baseline and follow-up } \\
\hline & & & & \multicolumn{3}{|c|}{ PD } & \multicolumn{3}{|c|}{ PSP } & \multicolumn{3}{|c|}{ CBD } \\
\hline & & & & Baseline & Follow-up & p & Baseline & Follow-up & p & Baseline & Follow-up & $\mathbf{p}$ \\
\hline ACE-R & 2 & 8.2 & $0.009^{*}$ & 90.1 & 88.0 & $0.04^{*}$ & 75.5 & 69.5 & 0.05 & 79.3 & 68.4 & $0.001^{*}$ \\
\hline MMSE & 2 & 8.0 & $0.007^{*}$ & 28.5 & 28.1 & 0.1 & 26.0 & 25.1 & ns & 26.0 & 25.1 & $0.004^{*}$ \\
\hline Attention/concentration & 2 & 6.3 & $0.04^{*}$ & 17.7 & 17.4 & ns & 16.4 & 15.5 & ns & 16.2 & 14.3 & ns \\
\hline Memory & 2 & 1.2 & ns & 21.5 & 21.6 & & 19.6 & 18.3 & & 19.5 & 18.9 & \\
\hline Fluency & 2 & 4.0 & ns & 10.8 & 10.3 & & 5.1 & 4.1 & & 8.3 & 6.5 & \\
\hline Language & 2 & 2.6 & ns & 24.9 & 24.0 & & 22.0 & 21.0 & & 23.1 & 20.4 & \\
\hline Visuospatial & 2 & 15.5 & $<0.0001^{*}$ & 15.3 & 14.8 & $0.003^{*}$ & 12.4 & 9.9 & $0.02 *$ & 12.1 & 8.3 & $0.0002^{*}$ \\
\hline Combined raw fluencies & 2 & 4.7 & ns & 29.5 & 29.6 & & 14.6 & 12.3 & & 23.4 & 18.9 & \\
\hline UPDRS III & & & & & & & 29.7 & 35.9 & ns & 29.1 & 36.2 & ns \\
\hline MDS-UPDRS III & & & & 30.5 & 41.0 & $<0.001^{*}$ & & & & & & \\
\hline
\end{tabular}


investigate the link between cognition and histological disease changes in more detail, and evaluating cognitive differentiation of atypical or intermediate syndromes which combine features of PD, PSP and CBD.

In principle, one could apply more detailed neuropsychological tests. However, an advantage of the ACE-R is ease of use, requiring short training for those administering it. Usually a full test will take around $10 \mathrm{~min}$ in cognitively well participants without akinesia, although it can be up to half an hour in severely affected patients with PSP and CBD. Verbal fluency is an even briefer test, taking just 2 min of testing time. The ACE-R and verbal fluency could be assessed routinely in a general neurology or movement disorders clinic and its utility has been shown in this study.

There are however several limitations to our study. The number of patients and follow-up time was limited. Furthermore, the duration of symptoms was shorter in the PD group, although correcting for this by comparing baseline PSP and CBD scores with follow-up PD scores led to the same pattern of results. Delayed diagnosis in PSP and CBD is common, with patients often receiving an alternative diagnosis for 1-2 years, therefore recruiting patients with PSP and CBD at an earlier stage is challenging. We cannot exclude the possibility that cognitive profiles would be different and between-group differences smaller at an earlier stage in the disease processes. Post-mortem confirmation of diagnosis was not available in all patients and so it is possible that for a small number of patients the diagnosis may be inaccurate. Patients with PD were seen for follow-up slightly later than the other two groups. However, correcting for this difference would augment rather than diminish our findings given the largest differences over time were seen in the CBD group who had the shortest mean follow-up.

Low scores may arise for different reasons in the three groups. For example, it is possible that apraxia or other features of a movement disorder impaired task performance differentially between groups, particularly on visuospatial drawing tasks and in patients with CBD. However, the majority of subjects were relatively early in the disease course, able to hold a pencil and draw sufficiently to reveal the cognitive elements of the test.

There are also caveats to our use of the UPDRS III motor subscale to measure motor severity. This scale does not capture all signs in the CBD phenotype, omitting apraxia, alien limb phenomena, dysarthrophonia and mirror movements. Although the principal axial and appendicular motor signs of PSP are included in the UPDRS III, it should be borne in mind that the test was developed for use in PD and that it may not be adequately balanced to sensitively grade the progressive severity of PSP or CBD. The use of the UPDRS should therefore be seen as descriptive and not diagnostic of our study populations.

\section{Concluding remarks}

We propose that the ACE-R score and verbal fluency are useful in supporting the differential diagnosis of parkinsonian disorders, including the sometimes difficult differentiation between PD and PSP. By the time of diagnosis (approximately 3 years from symptom onset for PSP), patients with PSP had lower ACE-R scores and severely reduced verbal fluency. The ACE-R may additionally be useful for measuring disease progression, especially in CBD, both in the clinical setting and in clinical trials of new therapies.

Acknowledgements We thank our patients and their families for supporting this work.
Contributors TR was responsible for experimental design, data collection, statistical analysis and manuscript preparation. BCG, DPB, JE and CW were responsible for experimental design, data collection and manuscript review. PM was responsible for data collection, statistical analysis and manuscript review. RAB was responsible for conception and experimental design, data collection and manuscript review. JBR was responsible for conception and experimental design, data collection, statistical analysis and manuscript preparation.

Funding This work was supported by the Medical Research Council grant number G1100464, the Wellcome Trust grant number 088324, and Parkinson's UK grant number J0801. Additional funding was obtained from the NIHR Cambridge

Biomedical Research Centre, including the Cambridge Brain Bank. DPB supported by Parkinson's UK/Big Lottery Fund. DPB, JRE, TR, CHWG and BG have been recipients of a Raymond and Beverly Sackler studentship. BG received funding from the Guarantors of Brain.

Competing interests None.

Ethics approval Cambridge 2 Research Ethics Committee.

Provenance and peer review Not commissioned; externally peer reviewed.

Open Access This is an Open Access article distributed in accordance with the Creative Commons Attribution Non Commercial (CC BY-NC 3.0) license, which permits others to distribute, remix, adapt, build upon this work non-commercially, and license their derivative works on different terms, provided the original work is properly cited and the use is non-commercial. See: http://creativecommons.org/ licenses/by-nc/3.0/

\section{REFERENCES}

1 Shaw LM, Korecka M, Clark CM, et al. Biomarkers of neurodegeneration for diagnosis and monitoring therapeutics. Nat Rev Drug Discov 2007;6:295-303.

2 Zheng Y, Cai T, Feng Z. Application of the time-dependent ROC curves for prognostic accuracy with multiple biomarkers. Biometrics 2006;62:279-87.

3 Williams-Gray C, Foltynie T, Brayne $C$, et al. Evolution of cognitive dysfunction in an incident Parkinson's disease cohort. Brain 2007;130:1787-98.

4 Ghosh BCP, Rowe JB, Calder AJ, et al. Emotion recognition in progressive supranuclear palsy. J Neurol Neurosurg Psychiatry 2009;80:1143-5.

5 Millar D, Griffiths P, Zermansky AJ, et al. Characterizing behavioral and cognitive dysexecutive changes in progressive supranuclear palsy. Mov Disord 2006:21:199-207.

6 Kertesz A, McMonagle P. Behavior and cognition in corticobasal degeneration and progressive supranuclear palsy. J Neurol Sci 2010;289:138-43.

7 Kompoliti K, Goetz CG, Boeve BF, et al. Clinical presentation and pharmacological therapy in corticobasal degeneration. Arch Neurol 1998;55:957-61.

8 Mathew R, Bak TH, Hodges JR. Diagnostic criteria for corticobasal syndrome: a comparative study. J Neurol Neurosurg Psychiatry 2011;83:405-10.

9 Aarsland D, Litvan I, Salmon D. Performance on the dementia rating scale in Parkinson's disease with dementia and dementia with Lewy bodies: comparison with progressive supranuclear palsy and Alzheimer's disease. I Neurol Neurosurg Psychiatry 2003;74:1215-21.

10 Pillon B, Blin J, Vidailhet $\mathrm{M}$, et al. The neuropsychological pattern of corticobasal degeneration: comparison with progressive supranuclear palsy and Alzheimer's disease. Neurology 1995;45:1477-83.

11 Bak TH, Rogers TT, Crawford LM, et al. Cognitive bedside assessment in atypical parkinsonian syndromes. J Neurol Neurosurg Psychiatry 2005;76:420-2.

12 Brenneis C, Seppi K, Schocke M, et al. Voxel based morphometry reveals a distinct pattern of frontal atrophy in progressive supranuclear palsy. I Neurol Neurosurg Psychiatry 2004; 75:246-9.

13 Price S, Paviour DC, Scahill R, et al. Voxel-based morphometry detects patterns of atrophy that help differentiate progressive supranuclear palsy and Parkinson's disease. Neuroimage 2004;23:663-9.

14 Josephs KA, Whitwell JL, Dickson DW, et al. Voxel-based morphometry in autopsy proven PSP and CBD. Neurobiol Aging 2008;29:280-9.

15 Boxer AL, Geschwind MD, Belfor N, et al. Patterns of brain atrophy that differentiate corticobasal degeneration syndrome from progressive supranuclear palsy. Arch Neurol 2006;63:81-6.

16 Mioshi E, Dawson K, Mitchell J, et al. The Addenbrooke's Cognitive Examination Revised (ACE-R): a brief cognitive test battery for dementia screening. Int J Geriatr Psychiatry 2006;21:1078-85.

17 Alexopoulos P, Ebert A, Richter-Schmidinger T, et al. Validation of the German revised Addenbrooke's Cognitive Examination for detecting mild cognitive impairment, mild dementia in Alzheimer's disease and frontotemporal lobar degeneration. Dement Geriatr Cogn Disord 2010;29:448-56.

18 Litvan I, Agid Y, Calne D, et al. Clinical research criteria for the diagnosis of progressive supranuclear palsy (Steele-Richardson-Olszewski syndrome): report of the NINDS-SPSP international workshop. Neurology 1996;47:1-9.

19 Robin X, Turck N, Hainard A, et al. PROC: an open-source package for R and S+ to analyze and compare ROC curves. BMC Bioinformatics 2011;12:77. 
20 Prince M, Bryce R, Ferri C, et al. World Alzheimer Report 2011: The Benefits of Early Diagnosis and Intervention. London: Alzheimer's Disease International, 2011.

21 García-Caballero A, García-Lado I, González-Hermida J, et al. Validation of the Spanish version of the Addenbrooke's Cognitive Examination in a rural community in Spain. Int J Geriatr Psychiatry 2006:21:239-45.

22 Yoshida H, Terada S, Honda H, et al. Validation of Addenbrooke's Cognitive Examination for detecting early dementia in a Japanese population. Psychiatry Res 2011;185:211-14

23 Henry JD, Crawford JR. Verbal fluency deficits in Parkinson's disease: a meta-analysis. J Int Neuropsychol Soc 2004;10:608-22.

24 Rosser A, Hodges JR. Initial letter and semantic category fluency in Alzheimer's disease, Huntington's disease, and progressive supranuclear palsy. J Neurol Neurosurg Psychiatry 1994;57:1389-94.

25 Graham NL, Bak TH, Patterson K, et al. Language function and dysfunction in corticobasal degeneration. Neurology 2003;61:493-9.

26 Shedlack KJ, Hunter R, Wyper D, et al. The pattern of cerebral activity underlying verbal fluency shown by split-dose single photon emission tomography (SPET or SPECT) in normal volunteers. Psychol Med 1991;21:687-96.

27 Heim S, Eickhoff SB, Amunts K. Different roles of cytoarchitectonic BA 44 and BA 45 in phonological and semantic verbal fluency as revealed by dynamic causal modelling. Neuroimage 2009:48:616-24.

28 Stuss DT, Alexander MP, Hamer L, et al. The effects of focal anterior and posterior brain lesions on verbal fluency. J Int Neuropsychol Soc 1998:4:265-78.

29 Dickson DW, Bergeron C, Chin SS, et al. Office of Rare Diseases neuropathologic criteria for corticobasal degeneration. J Neuropathol Exp Neurol 2002;61:935-46.

30 Dickson DW, Rademakers R, Hutton ML. Progressive supranuclear palsy: pathology and genetics. Brain Pathol 2007;17:74-82.
31 Braak $\mathrm{H}$, Tredici KD, Rüb U, et al. Staging of brain pathology related to sporadic Parkinson's disease. Neurobiol Aging 2003;24:197-211.

32 Shiner T, Seymour B, Wunderlich K, et al. Dopamine and performance in a reinforcement learning task: evidence from Parkinson's disease. Brain 2012:135:1871-83.

33 Bak TH, Caine D, Hearn VC, et al. Visuospatial functions in atypical parkinsonian syndromes. J Neurol Neurosurg Psychiatry 2006;77:454-6.

34 Clark CM, Sheppard L, Fillenbaum GG, et al. Variability in annual Mini-Mental State Examination score in patients with probable Alzheimer disease. Ann Neurol 1999:56:857-62.

35 McColgan P, Evans J. Addenbrooke's Cognitive Examination-Revised for mild cognitive impairment in Parkinson's disease. Mov Disord 2012;27: 1164-79.

36 Bensimon G, Ludolph A, Agid Y, et al. Riluzole treatment, survival and diagnostic criteria in Parkinson plus disorders: the NNIPPS study. Brain 2009;132:156-71.

37 Williams DR, de Silva R, Paviour DC, et al. Characteristics of two distinct clinical phenotypes in pathologically proven progressive supranuclear palsy: Richardson's syndrome and PSP-parkinsonism. Brain 2005;128:1247-58.

38 Evidente VGH, Adler $\mathrm{CH}$, Sabbagh MN, et al. Neuropathological findings of PSP in the elderly without clinical PSP: possible incidental PSP? Parkinsonism Relat Disord 2011;17:365-71.

39 Josephs KA, Petersen RC, Knopman DS, et al. Clinicopathologic analysis of frontotemporal and corticobasal degenerations and PSP. Neurology 2006:66:41-8.

40 Ling $\mathrm{H}, \mathrm{O}$ 'Sullivan $\mathrm{SS}$, Holton IL, et al. Does corticobasal degeneration exist? A clinicopathological re-evaluation. Brain 2010;133:2045-57. 